\title{
COMPUTER-AIDED DIAGNOSIS OF SOLID BREAST LESIONS USING AN ULTRASONIC MULTI-FEATURE ANALYSIS PROCEDURE
}

\author{
S. K. Alam ${ }^{1}$, E. J. Feleppa ${ }^{1}$, Mark Rondeau ${ }^{3}$, A. Kalisz ${ }^{1}$ and B. S. Garra ${ }^{2}$ \\ ${ }^{1}$ Riverside Research Institute, New York, NY, USA. \\ ${ }^{2}$ Food and Drug Administration, Silver Spring, MD, USA. \\ ${ }^{3}$ Self-similar Group, New York, NY, USA \\ email: kalam@ riversideresearch.org
}

\begin{abstract}
We have developed a family of quantitative descriptors in order to provide noninvasive, reliable means of distinguishing benign from malignant breast lesions. These include acoustic descriptors ("echogenicity," "heterogeneity," "shadowing") and morphometric descriptors ("area," "aspect ratio," "border irregularity," "margin definition"). These quantitative descriptors are designed to be independent of instrument properties and physician expertise. Our analysis included manual tracing of lesion boundaries and adjacent areas on grayscale images generated from RF data. To derive quantitative acoustic features, we computed spectral-parameter maps of radio-frequency (RF) echo signals (using a sliding-window Fourier analysis) of the lesion and adjacent areas. We quantified morphometric features by geometric and fractal analysis of traced lesion boundaries. Although no single parameter can reliably discriminate cancerous from non-cancerous breast lesions, multi-feature analysis provides excellent discrimination of cancerous and non-cancerous lesions. Our analysis of data acquired during routine ultrasonic examination of 130 biopsy-scheduled patients produced a receiver-operating characteristic (ROC) area under the curve (AUC) of 0.947 \pm 0.045 . Lesion-margin definition, spiculation, and border irregularity were the most useful among the quantitative descriptors; some morphometric features (such as border irregularity) also were particularly effective in lesion classification. Our results are consistent with many of the Breast Imaging Reporting and Data System (BI-RADS) breast-lesion-classification criteria in use today.
\end{abstract}

Keywords: Breast diseases, breast cancer, computer-aided diagnosis (CAD), fractal analysis, morphometric analysis, multi-feature analysis, receiver-operating characteristics (ROC), sonography, spectrum analysis, texture analysis, tissue characterization, tumor classification, ultrasonic imaging, ultrasound.

\section{BACKGROUND AND INTRODUCTION}

Breast cancer affects one of every eight women, it kills one of 29 women in the United States, and is the leading cause of death in women in developed countries [1,2]. An estimated 207,090 new cases of breast cancer, and 39,840 deaths, are expected among women in the US in 2010 [3]. Survival rates for advanced-stage breast cancers have improved significantly and early-stage breast cancers are now virtually curable [4]. Consequently, early detection can play a crucial role in a patient's survival.

Of the breast biopsies (annually around 1.7 million, according to National Cancer Institute estimate) performed in the US, 70-90\% are benign [5]. A method that reliably identifies benign lesions (with virtually zero false negatives) would prevent many unneeded biopsies, which are expensive and, as in any surgical procedure, involve minor risks. Assuming the average cost of a biopsy procedure to be $\$ 2,500$, even a 10\% reduction in biopsies (170,000 biopsies) would result in a saving of almost a half billion dollars a year in the US. (In fact, the more common surgical biopsies cost $\$ 2,500-\$ 5,000$, whereas needle biopsies cost $\$ 750-\$ 1,200$.) Furthermore, unneeded biopsies impose needless risk of complications, incur additional health-care costs, and needlessly heighten patient anxiety (e.g., while awaiting pathology results).

Unlike some other cancer types, most breast cancers are visible in B-mode ultrasound images. Advances in ultrasonic imaging technology allow detailed examination of breast-tumor characteristics. Although no single B-mode feature has been found to be a reliable identifier of malignancy, recent clinical studies have shown that a combination of selected B-mode features can be effective for breast cancer 


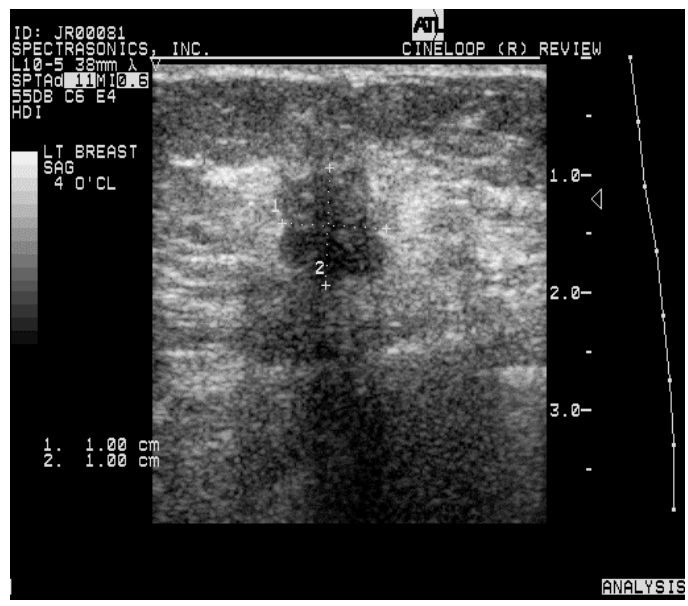

(a)

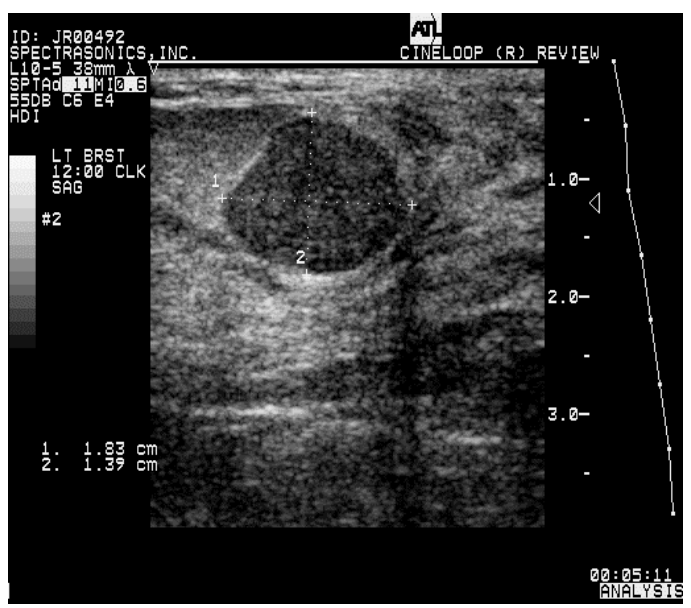

(b)

Fig. 1: (a) Malignant lesion (in situ and invasive ductal carcinoma): the lesion has irregular multilobular shape, "tall" aspect ratio, heterogeneous internal texture, poorly defined margin, and a prominent posterior shadow. (b) Benign lesion (fibroadenoma): the lesion has the classical near-spherical shape, a smooth boundary, clearly-defined margin, homogeneous internal texture, and a posterior "anti-shadow" or enhancement. (Note the edge shadows due to refractive effects.)

identification. [5,7-9] The American College of Radiology (ACR) developed the Breast Imaging Reporting and Data System (BI-RADS) lexicon for features describing the ultrasound appearance of breast lesions to improve the accuracy of breast ultrasound diagnosis [10,11]. BI-RADS defines six different possible findings (Category 0 to 5). Category 0 indicates that assessment is incomplete, additional imaging evaluation necessary, whereas Category 1 lesions are virtually certainly benign while Category 5 lesions have features that are highly suggestive of malignancy, i.e., the likelihood of malignancy increases from virtually zero in Category 1 to virtually certain in Category 5.

Several studies have reported encouraging results from automated quantitative analysis employing single [12-16] as well as multiple [17] features using data from modern ultrasonic scanners. This list is not exhaustive and many other groups reported results for automated methods of breast-cancer identification, although some studies ignored and did not compensate for the contribution from the ultrasound scanning system.

We implemented a quantitative multi-feature-analysis procedure that uses the BI-RADS criteria currently employed subjectively by clinicians using acoustic as well as morphometric features. The acoustic features include measures of lesion echogenicity, heterogeneity, and central shadowing, based on spectrum analysis of RF echoes [18]. The morphometric features include area, location, aspect ratio, and boundary roughness of the lesions. We employed hybrid features that use combined acoustic and border information, e.g., margin definition. Here we provide a brief report of our findings. We previously reported preliminary results for this study in conference proceedings $[19,20]$. We also published a detailed report of our findings in a journal paper [21].

\section{METHODS}

Diagnostically-useful lesion characteristics investigated in our study include features based on acoustic properties (acoustic features) as well as on their shapes or boundaries (morphometric features). The features found to be the most useful in the multi-feature studies are listed below in Table I. The following features are the most-important ones for distinguishing cancerous from non-cancerous lesions: internal texture (heterogeneous vs. homogeneous), central shadow (shadow vs. enhancement), shape (irregular vs. regular), aspect ratio (height divided by width) with respect to the duct axis (greater than unity vs. less than unity), border quality (irregular vs. regular), and margin definition (poorly defined vs. well defined). 
Figure 1 presents ultrasound grayscale image examples of a benign lesion and a malignant lesion with many of the typical characteristics of each lesion type. The malignant lesion exhibits heterogeneous internal texture, a central shadow, a poorly defined margin, an irregular shape, and a "tall" aspect ratio; all

Table I: Features of conventional B-mode images associated with malignant and benign lesions. A typical lesion may have only a subset of these identifying features.

\begin{tabular}{l|l|l|l}
\hline \multicolumn{2}{c|}{ Malignant lesions } & \multicolumn{2}{c}{ Benign lesions } \\
\hline \multicolumn{1}{c|}{ Internal features } & Morphometric features & \multicolumn{1}{c}{ Internal features } & Morphometric features \\
\hline Central shadowing & Irregular shape/spiculation & $\begin{array}{l}\text { Edge } \\
\text { shadowing/enhancement }\end{array}$ & Spherical/ovoid shape \\
\hline Hypoechogenicity & Poorly defined margin & Hyperechogenicity & Linear well-defined margin \\
\hline Heterogeneous texture & Tall aspect ratio & Homogeneous texture & Thin capsule \\
\hline Calcifications & Microlobulation & & Gentle bi- or trilobulations \\
\hline & Architectural distortion & & $\begin{array}{l}\text { Orientation parallel to tissue } \\
\text { plane }\end{array}$ \\
\hline
\end{tabular}

these features are typical of malignant lesions. In contrast, the benign lesion exhibits a central posterior enhancement (sometimes referred to as "anti-shadow"), a homogeneous internal texture, a clearly defined margin, a smooth shape, and an aspect ratio of less than unity typical of benign lesions. However, both lesions are hypoechoic.

Successful classification using these qualitative image characteristics is invariably dependent on clinician skills. Our research addresses the development of quantitative descriptors to provide operator-independent lesion identification. Quantitative descriptors will also increase reliability of lesion identification and may allow identification of smaller lesions. We first identified features that lend themselves to quantification; not all subjective features are reliably quantifiable. The procedure to quantify acoustic and morphometric features is described below.

\subsection{Data Acquisition}

The data for this IRB-approved study consisted of RF echo-signal data that were digitized from breast lesions before any non-linear processing such as compression or envelope detection occurred within the scanner. These data were acquired from 130 patients during routine ultrasonic examinations that occurred prior to a scheduled biopsy. Subsequent biopsies of examined lesions determined that 104 patients had benign masses and 26 had malignant masses. These biopsy and RF data were acquired at the following three clinical sites: Thomas Jefferson University, the University of Cincinnati, and Yale University. These patients had undergone mammography prior to the ultrasound examinations and had mammographically visible lesions. The following exclusion criteria were applied: age less than 18 years (due to legal consent limitations), prior breast carcinoma, biopsy or mastectomy, breast implant, simple cyst, pregnancy, microcalcifications not associated with a mass on sonography, and male or transsexual gender. Masses were examined with the patient in the standard supine position by an experienced radiologist or sonographer using a Philips Ultrasound (Bothell, WA) UM-9 HDI scanner. An L10-5 (7.5 MHz) linear array transducer was employed at a default (constant) power level and a single transmit focal length selected by the operator. Standard ultrasonic breast-examination procedures were employed. Data were sampled at $20 \mathrm{MHz}$ at an effective dynamic range of 14 bits. Time Gain Control (TGC) data were acquired for every scan, and RF data were corrected for TGC before processing. 


\subsection{Analysis Procedures}

We manually demarcated a set of analysis regions on B-mode images generated from the RF data and analyzed each region to compute the quantitative features described below. The benign and malignant lesions of Fig. 1 are shown again in Fig. 2 with traces of the nine analysis regions superimposed. With respect to the tumor, these regions are: left-anterior, tumoranterior, right-anterior, left-lateral, tumor, right-lateral, left-posterior, tumor-posterior, and right-

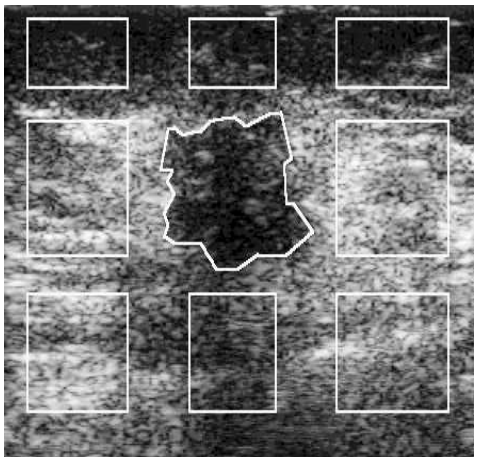

(a)

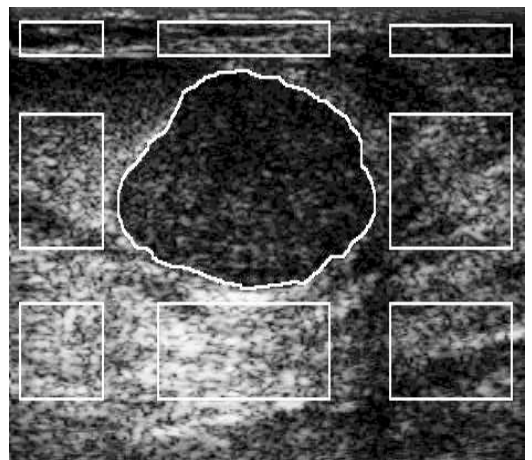

(b)

Fig. 2: B-scan images of Fig. 1 with superimposed analysis-region traces.

(a) Malignant lesion. (b) Benign lesion. posterior. As discussed below, we needed only the tumor trace for the majority of quantitative features. However, we needed the tumor-posterior region as well as the left and right-posterior regions for shadow measurements and all analysis regions except the anterior regions for computing relative absorption. Most lesions were scanned in multiple (but at least two orthogonal) scan planes, thereby providing redundant data for a given lesion. We averaged each quantitative feature value for multiple scans of a specific lesion to arrive at a single number. All processing software was written in MATLAB ${ }^{\mathrm{TM}}$ (The Mathworks, Inc., Natick, MA), except for the tumor-tracing program, which was written in Visual Basic ${ }^{\mathrm{TM}}$ (Microsoft Corporation, Redmond, WA).

\subsection{Acoustic Features}

We defined quantitative acoustic features in terms of calibrated spectrum analysis [18] parameters. Spectrum analysis involves several steps. First, a Hamming window is applied to RF data, a power spectrum is computed from the Fourier transform of the windowed data segment, and the resultant power spectrum is converted to dB. Next, system and diffraction effects ${ }^{\dagger}$ are subtracted from the computed spectrum to derive the desired tissue spectrum. Finally, the

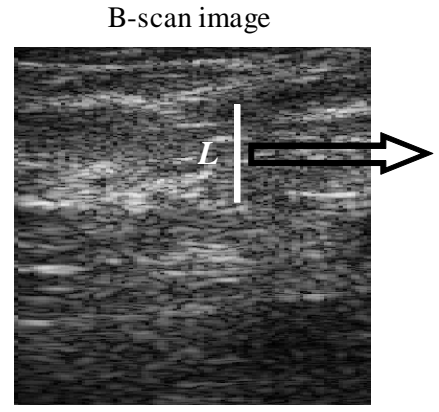

(a)

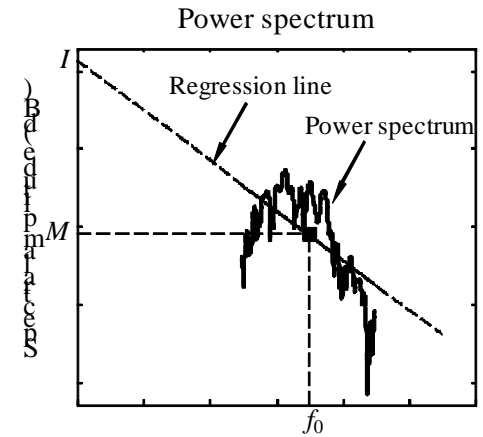

Frequency (MHz)

(b)

Fig. 3: Illustration of the Spectrum Analysis procedure. Calibrated power spectrum of windowed (typically Hamming, of length $L$ ) RF data is evaluated. A linear regression line through the power spectrum is computed. In this example, $M$ is the midband value (value of the regression line at center frequency $f_{0}$ ) and $I$ is the spectral intercept (value of the regression line extrapolated to $f=0$ ).

\footnotetext{
$\dagger$ Measured spectra depend not only on tissue properties, but also on 1) the combined two-way transfer function of the transducer and the ultrasonic-system electronic modules, 2) the two-way range-dependent diffraction function (beam properties), and 3) acoustic attenuation. Corrections for the first two functions involve experimental data obtained at each transmit focal length. The electronic transfer function was estimated using a planar reflection method from RF data acquired from the front planar surface of an RTV silicone block in a water bath. The diffraction function was estimated using a reference phantom method from data obtained by scanning a rubber block containing a diffuse suspension of $10-\mu \mathrm{m}$ diameter glass spheres. Then, breast tissue spectral parameters were estimated by subtracting the contribution from transfer function and diffraction. Finally, an empirical attenuation coefficient was used to correct for attenuation as described further below. The system effects are analyzed in detail in other theoretical papers $[18,23,24]$.
} 
computed spectrum is analyzed with linear regression techniques applied over the bandwidth of the signal; the primary parameters of interest are the slope of the regression line (SLP, $s$ ), its value at midpoint of signal bandwidth (MBF, $M$ ) and its intercept at zero frequency (INT, $I$ ). Images of these parameters are created by progressively sliding the Hamming window over all RF data and repeating the above sequence. The spectrum analysis procedure is illustrated in Fig. 3.

In the absence of attenuation in the intervening media, the linear regression line through the power spectrum can be written as, $P(f)=I+s f$, where $I$ is spectral intercept, $m$ is slope, and $f$ is frequency. Thus, the midband fit, $M=I+s f_{0}, f_{0}$ being the center frequency. In the presence of attenuation in intervening tissue, the linear regression line through the power spectrum is $P_{\alpha}(f)=P(f)-2 \alpha d f=I+(s-$ $2 \alpha d) f$, where $\alpha$ is the effective attenuation coefficient $(\mathrm{dB} / \mathrm{MHz}-\mathrm{cm})$ and $d$ is the depth of intervening tissue. Hence, spectral intercept $I_{\alpha}=I$, midband fit $M_{\alpha}=I+(s-2 \alpha d) f_{0}$, and slope $s_{\alpha}=(s-2 \alpha d)$. Thus, the presence of attenuation affects slope and midband fit, but not intercept. The necessary assumption is

(a) B-scan image

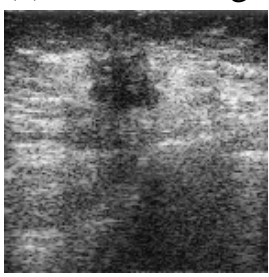

(c) I within lesion

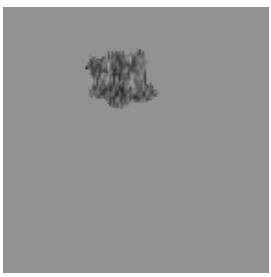

(b) Image of $I$

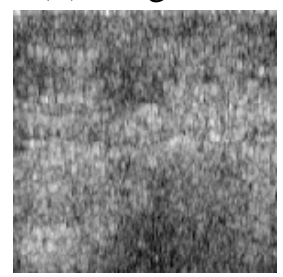

(d) Histogram of $I$

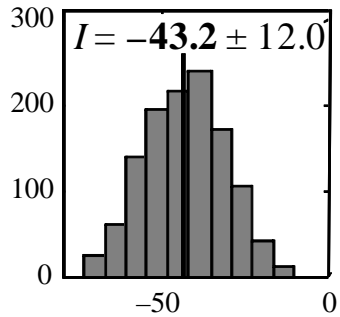

(a) B-scan image

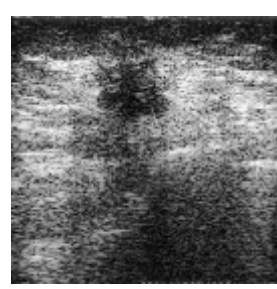

(c) $M$ in the ROIs

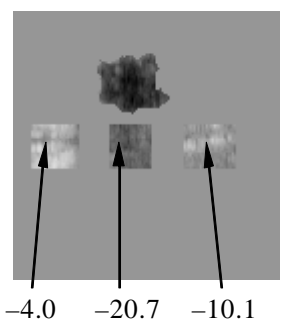

(b) Image of $M$

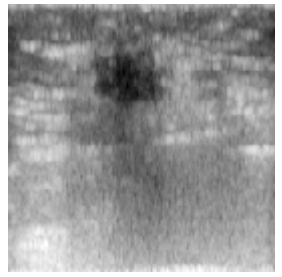

(d) Shadowing effect

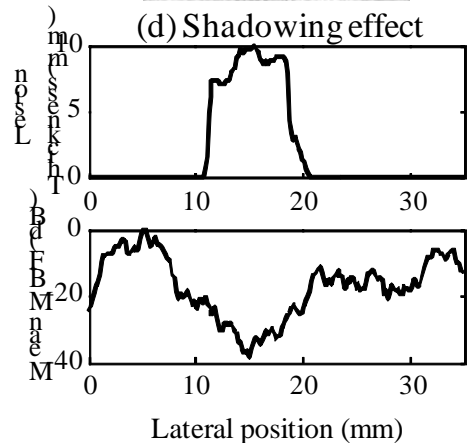

Fig. 4: Echogenicity for data corresponding to Fig. 3(a). According to our definition, echogenicity is $-43.2 \mathrm{~dB}$.

Fig. 5: Shadowing for data corresponding to Fig. 3(a). According to our definition, shadowing is $-13.65 \mathrm{~dB}$.

that attenuation (in $\mathrm{dB}$ ) varies linearly with frequency. Although this assumption is only approximate, the conclusion about the invariance of intercept in the presence of tissue attenuation has proved to be accurate in our experience.

In our work, the following definitions are employed to provide quantitative assays qualitative (B-mode) acoustic features: window length, $W=2.4 \mathrm{~mm}$, spectral bandwidth, $B=4 \mathrm{MHz}$ (5-9 MHz), and attenuation coefficient, $\alpha=1 \mathrm{~dB} / \mathrm{MHz}-\mathrm{cm}$.

A) Echogenicity is defined as the mean value of spectral intercept, $\mu_{I_{L}}$, within the lesion. Since spectral intercept is largely independent of frequency-dependent attenuation in the intervening media, no attenuation correction is necessary. Calculation of lesion echogenicity is illustrated in Fig. 4 using the example of invasive ductal carcinoma shown in Fig. 1a. Figure 4a shows the B-scan image; the corresponding image of spectral intercept $(I)$ is shown in Fig. 4b. Figure 4c shows the intercept image within the traced lesion boundary and Fig. 4d shows the histogram of $I$ within the traced lesion. The quantitative value of echogenicity is the mean value of INT within the lesion $(-43.2 \mathrm{~dB})$. The 
fibroadenoma in Fig. 1b has an echogenicity of $-36.0 \mathrm{~dB}$.Shadowing is defined as the difference (normalized by lesion thickness) between mean $M$ values in comparable shadowed and unshadowed regions posterior to the lesion. (Difference between tumor and tumor-posterior is compared with the average of differences between left-lateral and left-posterior, and right-lateral and right-posterior.) This difference can be used to estimate the attenuation coefficient within the lesion, which we used in the tissue area that has no lesion to estimate the average attenuation coefficient and check whether these estimates are consistent with the assumed value. Calculation of central shadowing is illustrated in Fig. 5 for the cancerous lesion in Fig. 1a, casting a noticeable central shadow. Figure 5a shows the B-scan image; the corresponding image of $M$ is shown in Fig. 5b. Figure 5c shows the MBF image inside the tumor as well as in the posterior regions. Mean values of $\mathrm{MBF}$ in the lateral posterior regions are -4.0 and $-10.1 \mathrm{~dB}$ (their mean being $-7.05 \mathrm{~dB}$ ). The mean of MBF posterior to the lesion is $-20.7 \mathrm{~dB}$, which is $13.65 \mathrm{~dB}$ lower. Thus, this tumor casts a $13.65-\mathrm{dB}$ central shadow. Figure $5 \mathrm{~d}$ separately plots the vertical thickness of the tumor and mean-value of $M$ in the posterior region vs. lateral position. The mean-value of $M$ decreases with increasing lesion thickness and is the lowest at the thickest point of the tumor. The fibroadenoma in Fig. 1b has caused a central posterior enhancement of $+8.35 \mathrm{~dB}$.

B) Relative absorption is a composite feature and is defined as $r a=\left(M_{p n}-M_{a n}\right) / d_{1}-\left(M_{p l}-M_{a l}\right) / d_{2}$ [22], where $M_{a l}$ is the mean of midband fit inside the lesion, $M_{p l}$ is the mean of midband fit posterior to lesion, $M_{a n}$ is the mean of midband fit in normal tissue next to the lesion, $M_{p n}$ is the mean of midband fit in normal tissue lateral posterior to next to the lesion, $d_{1}$ is the spatial distance between the centroids of $M_{p n}$ and $M_{a n}$, and $d_{2}$ is the spatial distance between the centroids of $M_{p l} ; M_{a l}, M_{p n}$, and $M_{a n}$ can be averaged for left and right lateral regions. Relative absorption value for the malignant lesion in Fig. 1a is $-0.12 \mathrm{~dB}$. In contrast, the fibroadenoma in Fig. $1 \mathrm{~b}$ has a relative absorption of $-0.47 \mathrm{~dB}$.

C) We can define heterogeneity in several ways. It can be defined as the standard deviation of midband fit values, $\sigma_{M_{L}}$, within the lesion [23] and we can assess the heterogeneity of the lesion by comparing $\sigma_{M_{L}}$ with $\sigma_{M}$ for a homogeneous region. In homogeneous tissue regions, the standard deviations of $M, s$, and $I$, can be expressed as [23] $\sigma_{M}=5.6 / \sqrt{B W}, \sigma_{s}=5.6 \sqrt{12} /(B \sqrt{B W})$, and $\sigma_{I}=\sqrt{\sigma_{M}^{2}+f_{0} \sigma_{s}^{2}}$, respectively, where $f_{0}$ is the center frequency (MHz), $B$ is the bandwidth (MHz), and $W$ is the Hamming-window length (mm). As tissue becomes more heterogeneous, the standard deviations of these measured parameters increase from the above theoretical values. We select $\sigma_{M}$ to provide an index of tissue heterogeneity because $M$ typically provides less-noisy estimates compared to the other two spectral parameters; this permits smaller departures from homogeneity to be detected. The standard deviation of MBF inside the lesion for the invasive ductal carcinoma in situ in Fig. 1a is $5.6 \mathrm{~dB}$, whereas it should be $1.77 \mathrm{~dB}$ for a homogeneous region. (For our processing parameters, $L=2.5 \mathrm{~mm}, f_{0}=7.5 \mathrm{MHz}$, and $B=4 \mathrm{MHz}, \sigma_{M}=1.77, \sigma_{s}=1.53$, and $\sigma_{I}=4.55$.) In contrast, $\sigma_{M}$ for the fibroadenoma in Fig. $1 \mathrm{~b}$ is $4.4 \mathrm{~dB}$.

Heterogeneity also may depend on texture and $\sigma_{M}$ contains no textural information. Therefore, we defined heterogeneity in term of texture of midband fit inside the lesion; texture was defined in terms of a fourneighborhood pixel algorithm [25] (FNPA) and Hurst Coefficient fractal dimension measure [26,27]. (The cooccurrence matrix has also been used to estimate B-mode texture [28], but the calculation cost of the co-occurrence matrix is high.) FNPA yields $-0.03 \mathrm{~dB}$ for the cancer in Fig. $1 \mathrm{a}$ and $-0.04 \mathrm{~dB}$ for the lesion in Fig. 1b.

\subsection{Morphometric Features}

Invasive ductal carcinomas generally have "fuzzy" borders due to their invasive margins. Cancers that have little desmoplastic reaction (proliferation of fibroblasts) typically have clear margins, but still are highly irregular in shape. Chou et al [15] demonstrated good performance (97.2\% sensitivity and $94.1 \%$ negative predictive value) using only quantitative lesion-shape features describing irregular boundaries (or deviation from a smooth shape). We have employed the following definitions for the quantitative 
morphometric descriptors that are related to the shape or border of the lesion. All morphometric features have been computed using lesion boundaries traced on B-mode images.

A) Area is defined as the total lesion area in square $\mathrm{cm}$. Lesion area for the cancer in Fig. 1a is $0.73 \mathrm{~cm}^{2}$. Lesion area has not been found to be a reliable feature for lesion classification.

B) Aspect Ratio is defined as the maximum vertical lesion-dimension divided by maximum horizontal lesion-dimension. The aspect ratio (height divided by width) often exceeds 0.8 in breast carcinomas for small lesions [29]. In larger carcinomas, this criterion is less useful due to their more irregular shapes and growth along duct axes. The lesion aspect ratio is 0.97 for the cancer in Fig. 1a and 0.75 for the benign lesion (smaller) in Fig. $1 \mathrm{~b}$.

C) We define border irregularity in terms of a fractal dimension. Fractal dimension of a closed contour can be used to represent its border roughness [30]. Mandelbrot [31] has investigated the fractal dimensions of geographical boundaries. On the other hand, convexity [32] (ratio between convex perimeter and actual lesion perimeter) also can be used to express border irregularity; this is an excellent descriptor of spiculation. We illustrate using four contours of varying border roughness in Fig. 6. The smooth ovoid in Fig. 6a has a low fractal dimension (1.01) and convexity of unity. For the non-spiculative rough border depicted in Fig. $6 \mathrm{~b}$, fractal dimension is 1.05 and convexity is 0.86 . For the mild spiculation in Fig. 6c, fractal dimension is 1.06 whereas convexity is 0.84 . For the moderate spiculation in Fig. 6d, the fractal dimension is higher (1.18) and the convexity is much lower $(0.51)$. We note that further increase in spiculation drastically reduces convexity; thus, convexity is rather adept at identifying spiculation and also an excellent descriptor of border irregularity. Fractal dimension is also an excellent quantitative descriptor of border irregularity. For the malignant lesion in Fig. 1a, the fractal dimension and convexity are 1.13 and 0.90 , respectively, and for the benign lesion in Fig. 1b, they are 1.03 (lower) and 0.99 (higher), respectively.

D) We have defined margin definition as the sum of magnitude of gradient of midband fit on a lesion contour normalized by the sum of magnitude of the gradient of midband fit on the lesion contour. (Normalization is required to remove dependence on contour length as well as magnitudes of midband fit values.) Because this feature uses both the lesion contour as well as a spectral parameter, it really is a hybrid feature. We use midband fit instead of envelope of RF echoes because MBF is statistically wellbehaved and can more easily be corrected for system effects and diffraction. Gradient-based margin definition for the lesions in Figs. 1a and $1 \mathrm{~b}$ are 0.07 and 0.10 , respectively.

\section{RESULTS}

We have analyzed data for 130 patients $(26$ malignant and 104 benign). Scatter diagrams for selected quantitative acoustic features and morphometric features are presented in Fig. 7. The scatter diagrams of Fig. 7a (margin definition vs. aspect ratio)

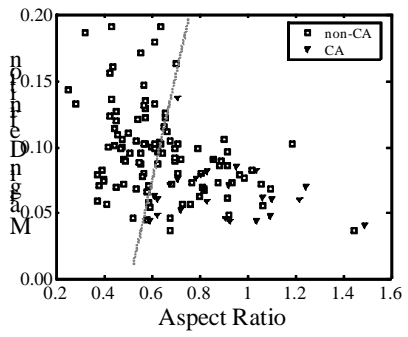

(a)

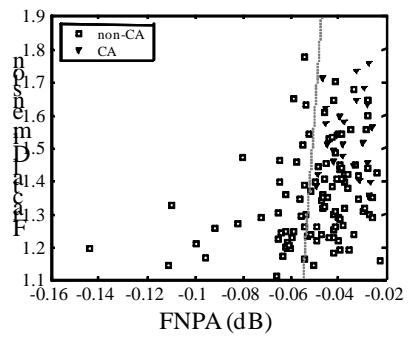

(b)
Fig. 7: Scatter diagrams of selected lesion features. 
and $7 \mathrm{~b}$ (fractal dimension $v s$. lesion texture) exhibit fairly clear separation between benign and malignant cases. With respect to non-cancers, the cancer cases exhibit poorer margin definition, larger aspect ratio, higher texture, and higher border irregularity. We drew a straight line through each scatter diagram such that all the malignant lesions are on the right of the lesion. Thus, there only are benign lesions on the left of each line. If we want to be even more conservative and further reduce the possibility of a false negative, we could more the line more to the left.

We performed an independent-samples t-test to assess whether the means of different parameters are statistically different for benign and malignant cases. We found that FNPA, Hurst Coefficient, Margin Definition, Aspect Ratio, Solidity, Convexity, and Hausdorf Dimension are significantly different for benign and malignant cases. Additional details about the t-test are in [21]. Because these features show fairly clear separation between cancers and non cancers, a linear classification approach is indicated. We used logistic regression (LR) for our classification analysis. All statistical analysis were performed using SPSS $^{\circledR}$ (SPSS Inc., Chicago, IL) using all quantitative features. Out of 130 patients, 121 were used; the other 9 had at least one quantitative feature missing. (For example, shadowing cannot be computed if posterior regions cannot be traced.) The classifier incorporated heterogeneity, margin definition, fractal dimension, and convexity using Wilks' Lambda Stepwise Statistics.

Classification performance was assessed using an ROC analysis33] ], which plotted true-positive fraction (TPF) or sensitivity $v s$. false-positive fraction (FPF) or 1 minus specificity. TPF and FPF are defined as:

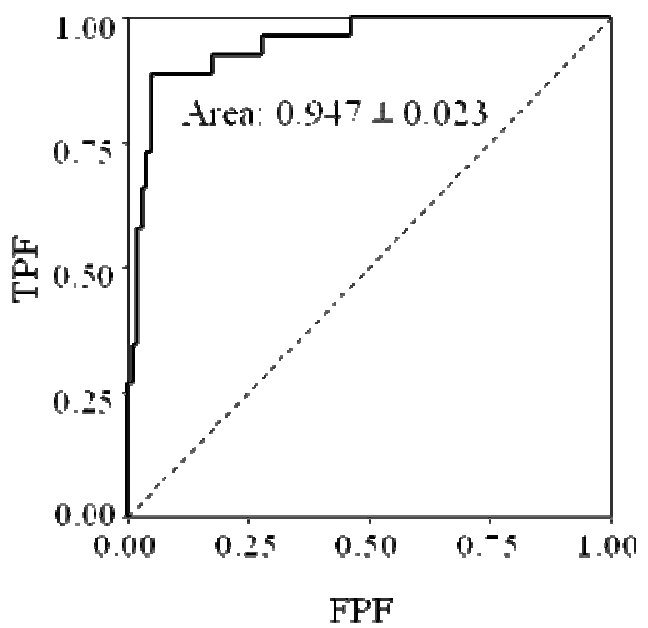

Fig. 8: ROC curve for multi-feature analysis. ROC Area: $0.9164 \pm 0.0346$.

$$
\begin{aligned}
& T P F=\frac{\text { Number of correctly identified malignant lesions }}{\text { Total number of malignant lesions }} \text { and } \\
& F P F=1-\frac{\text { Number of correctly identified benign lesions }}{\text { Total number of benign lesions }}
\end{aligned}
$$

Incorporation of all four parameters in an LR produced an area under the ROC curve (AUC) of $0.947 \pm 0.045$ (Fig. 8). We do not report sensitivity or specificity because these depend on the chosen operating point in the ROC curve. Best methods achieve high TPF values, i.e., high sensitivity values, for concurrently low FPF values, i.e., high specificity values.

\section{CONCLUSION}

Many radiologists now use breast ultrasound findings based in BI-RADS criteria, to recommend periodic follow-up without a biopsy. Whereas an expert clinician might be able to identify benign lesions accurately, a non-expert might misidentify ambiguous cases. We studied the performance of classification using quantitative features to determine whether such an approach might be of value in reducing misdiagnoses by less-expert readers. Our results suggest that an automated or semi-automated procedure might be able to assist a physician in making diagnosis. Clearly, the method is not foolproof (ROC Area $\neq 1.0$ ). Due to the current legal environment in the western countries (particularly in the US), the radiologists tend to be conservative. Our results suggest that we can achieve a near-perfect NPV (negative-predictive value) if we design the procedure to be conservative by operating in the low FPF area (e.g., where $1 / 4 \leq \mathrm{TPF} \leq 1 / 2$ ). This, 
however, will mean a decreased number of avoided biopsies. The current procedure remains semiquantitative as analysis regions are manually traced. Our recent work on automated boundary detection suggests that this method can be truly operator independent with automated lesion boundary segmentation. Note that manual lesion segmentation was performed by knowledgeable non-clinicians in our study. Thus, the method does not depend on a radiologist's expertise for precise delineation of tumor borders. We will investigate the role of additional factors e.g., age, body-mass index or equivalent, etc. Furthermore, breast composition varies from person to person, which seems likely to influence RF echoes. Thus, for acoustic features in particular, the presumably normal, opposite (contralateral) breast tissue may provide a baseline to compare with the lesion-containing breast [34]. Because tissue properties change with time and differ among patients, such an approach would compensate for variations in breast density, time of the menstrual cycle, changes occurring at menopause, fat content, age, etc. in future analyses. Furthermore, our study data did not distinguish among fibrous, glandular, and fatty breasts. This may be important because breast composition can affect shadow values.

Because their breasts typically are radiologically-dense, X-ray mammography tends to be relatively ineffective for young women. The sensitivity of X-ray mammography is significantly less $(60 \%)$ in younger women (less than 50 years old) compared to women older than 50 (86\%) [35]. However, breast tumors tend to grow faster in younger, estrogen-rich women [36]. Thus, early detection may be even more critical for the survival of younger women with breast cancer, where ultrasound can play an important role.

\section{ACKNOWLEDGMENTS}

This work was supported in part by US Army Medical Research and Material Command grant DAMD1798-1-8331 and Susan G. Komen for the Cure grant KG081601. Data used in this study were acquired at the University of Cincinnati, Thomas Jefferson University, and Yale University. We are indebted to our late colleague Dr. Frederic Lizzi for his inspirational support and advice with the analysis. Ms. Rumana Huq and Ms. Stella Urban traced lesions using in-house software.

\section{REFERENCES}

1. P. Pisani, D. M. Parkin, and J. Ferlay, "Estimates of the worldwide mortality from eighteen major cancers in 1985. Implications for prevention and projections of future burden," Int. J. Cancer, vol. 55, pp. 891-903, 1993.

2. L. Ries, L. Kosary, B. Hankey, B. Miller, B. Edwards, SEER Cancer Statistics Review, 1973-1996, National Cancer Institute, Bethesda, MD, 1999.

3. American Cancer Society, Cancer Facts \& Figures, 2010.

4. F. A. Vicini, L. Kestin, P. Chen, P. Benitez, N. S. Goldstein, and A. Martinez, "Limited-Field Radiation Therapy in the Management of Early-Stage Breast Cancer," J National Cancer Institute, vol. 95, pp. 1205-1210, 2003.

5. A. T. Stavros, D. Thickman, C. L. Rapp, M. A. Dennis, S. H. Parker, and G. A. Sisney, "Solid breast nodules: use of sonography to distinguish between benign and malignant lesions," Radiology, vol. 196, pp. 123-134, 1995.

6. C. Cole-Beuglet, R. Z. Soriano, A. B. Kortz, B. B. Goldberg, "Ultrasound analysis of 104 primary breast carcinomas classified according to histologic type," Radiology, vol. 147, pp. 191-196, 1983.

7. Advanced Technology Laboratories, "Summary of ATL-sponsored breast ultrasound study and preliminary findings," ATL publications, 1994.

8. P. Skaane and K. Engedal, "Analysis of sonographic features in the differentiation of fibroadenoma and invasive ductal carcinoma," AJR (American Journal of Roentgenology), vol. 170, pp. 109-114, 1998.

9. S. Huber, J. Danes, I. Zuna, J. Teubner, M. Medl, and S. Delorme, "Relevance of sonographic B-mode criteria and computer-aided ultrasonic tissue characterization in differential/diagnosis of solid breast masses," Ultrasound Med Bio., vol. 26, pp. 1243-1252, 2000.

10.ACR Breast Imaging Reporting and Data System (BI-RADS) Atlas, American College of Radiology, Reston, VA, 2003.

11.E. B. Mandelson, N. A. Berg, C. R. B. Merritt, "Towards a Standardized Breast Ultrasound Lexicon BI-RADS: Ultrasound," Semin Roentgenol, vol. 36, pp. 217-25, 2001. 
12.K. D. Donohue, L. Huang, T. Burks, F. Forsberg, and C. W. Piccoli, "Tissue classification with generalized spectrum parameters," Ultrasound Med. Biol., vol. 27, no. 11, pp. 1505-1514, 2001.

13.P. M. Shankar, V. A. Dumane, J. M. Reid, V. Genis, F. Forsberg, C. W. Piccoli, and B. B. Goldberg, "Classification of Ultrasonic B-Mode Images of Breast Masses Using Nakagami Distribution," IEEE Trans. Ultrason. Ferroel. Freq. Contr., vol. 48, pp. 569-580, 2001.

14.D.-R. Chen, R.-F. Chang, and Y.-L. Huang, "Breast cancer diagnosis using self organizing map for sonography," Ultrasound Med. Biol., vol. 26, pp. 405-411, 2000.

15.Y.-H. Chou, C.-M. Tiu, G.-S. Hung, S.-C. Wu, T. Y. Chang, and H. K. Chiang, "Stepwise logistic regression analysis of tumor contour features for breast ultrasound diagnosis," Ultrasound Med. Biol., Vol. 27, No. 11, pp. 1493-1498, 2001.

16. Y. Zheng, J. F. Greenleaf, and J. J. Gisvold, "Reduction of breast biopsies with a modified self-normalizing map," IEEE Trans. Neural Networks, vol. 8, pp. 1386-1396, 1997.

17.F. Lefebvre, M. Meunier, F. Thibault, P. Laugier, and G. Berger, "Computerized ultrasound B-scan characterization of breast nodules," Ultrasound Med. Biol., vol. 26, pp. 1421-1428, 2000.

18.F. L. Lizzi, M. Greenebaum, E. J. Feleppa, M. Elbaum, and D. J. Coleman, "Theoretical framework for spectrum analysis in ultrasonic tissue characterization," J Acoust Soc Am, vol. 73, pp. 1366-1373, 1983.

19.S. K. Alam, F. L. Lizzi, E. J. Feleppa, T. Liu, and A. Kalisz, "Ultrasonic multi-feature analysis procedures for breast lesion classification," Proceedings of SPIE 3982, Medical Imaging 2000: Ultrasonic Imaging and Signal Processing, K. Kirk Shung, Michael F. Insana, Editors, pp. 196-201, 2000.

20. S. K. Alam, F. L. Lizzi, E. J. Feleppa, T. Liu, and A. Kalisz, "Computer aided diagnosis of breast lesions using a multi-feature analysis procedure," Proceedings of SPIE 4687, Medical Imaging 2002: Ultrasonic Imaging and Signal Processing, Michael F. Insana, W. Walker, Editors, pp. 296-303, 2002.

21.S. K. Alam, E. J. Feleppa, M. Rondeau, A. Kalisz, and B. S. Garra, "An ultrasonic multi-feature analysis procedure for computer-aided diagnosis of solid breast lesions," Ultrason Imag, 2010 (In Press).

22.C. B. Shakespeare, personal communication, 1998.

23.F. L. Lizzi, E. J. Feleppa, M. Astor, and A. Kalisz, "Statistics of ultrasonic spectral parameters for prostate and liver examinations," IEEE Trans. Ultrason., Ferroelect., Freq. Contr., vol. 44, pp. 935-942, 1997.

24.FL Lizzi, SK Alam, S Mikaelian, P Lee, and EJ Feleppa, "On the statistics of ultrasonic spectral parameters," Ultrasound Med Biol, vol. 32, pp. 1671-1685, 2006.

25.W. Yao, B. Zhao, Y. Zhao, W. Wang, and G. Qian, "Ultrasonographic texture analysis of parenchymatous organs by the four-neighborhood-pixels algorithm," J. Ultrasound Med., vol. 20, pp. 465-471, 2001.

26.H. E. Hurst, R. P. Black, Y. M. Simaika, Long term storage: an experimental study, Constable, London, 1965.

27.J. Feder, Fractals, Plenum Press, New York, 1988.

28. B. S. Garra, B. H. Krasner, S. C. Horii, S. Ascher, S. K. Muk, and R. K. Zeman, "Improving the distinction between benign and malignant breast lesions: the value of sonographic texture analysis," Ultrason. Imag., vol. 13, pp. 267-285, 1993.

29. E. Tohno, D. O. Cosgrove, and J. P. Sloane, Ultrasound Diagnosis of Breast Diseases, Churchill Livingstone, London, UK, 1994.

30. L. F. Richardson, "The problem of contiguity: An appendix of statistics of deadly quarrels," General Systems Yearbook, vol. 6, pp. 139-187, 1961.

31. B. B. Mandelbrot, "How long is the coast of britain? statistical self-similarity and fractional dimension," Science, vol. 155, pp. 636-638, 1967.

32.J. C. Russ, The Image Processing Handbook, CRC Press, Boca Raton, FL, 1999.

33.C. E. Metz, J. H. Shen, and B. A. Herman, "New methods for estimating a binormal ROC curve from continuously-distributed test results," Presented at the 1990 Joint Meetings of the American Statistical Society and the Biometric Society, Anaheim, CA, August 1990.

34. S. S. Kaplan, "Clinical Utility of Bilateral Whole-Breast US in the Evaluation of Women with Dense Breast Tissue," Radiology, vol. 221, pp. 641-649, 2001.

35.L. Tabàr, G. Fagerberg, S. W. Duffy, N. E. Day, A. Gad, and O. Gröntoft, "Update of the Swedish two-county program of mammographic screening of breast cancer," Radiol Clin North Am, vol. 30, pp. 187-210, 1992.

36.P. G. M. Peer, J. A. A. M. van Dijck, J. H. C. L. Hendricks, R. Holland, A. L. M. Verbeek, "Age-dependent growth rate of primary breast cancer," Cancer, vol. 71, pp. 3547-3551, 1993. 